PROCEEDINGS OF THE

AMERICAN MATHEMATICAL SOCIETY

Volume 136, Number 10, October 2008, Pages 3717-3726

S 0002-9939(08)09359-3

Article electronically published on May 19, 2008

\title{
DISCRETE CONNECTION LAPLACIANS
}

\author{
SVETOSLAV ZAHARIEV
}

(Communicated by Mikhail Shubin)

\begin{abstract}
To every Hermitian vector bundle with connection over a compact Riemannian manifold $M$ one can associate a corresponding connection Laplacian acting on the sections of the bundle. We define analogous combinatorial, metric dependent Laplacians associated to triangulations of $M$ and prove that their spectra converge, as the mesh of the triangulations approaches zero, to the spectrum of the connection Laplacian.
\end{abstract}

\section{INTRODUCTION}

Let $M$ denote a compact Riemannian manifold. Combinatorial analogues of the Laplacian on $M$ have been considered in various contexts since the appearance of 9. In the 1970s Dodziuk defined combinatorial, metric dependent Laplacians associated to triangulations of $M$ and proved that under certain technical assumptions the spectra of these operators converge to the spectrum of the Laplacian acting on functions on $M$ as the mesh of the triangulations tend to zero; cf. [6]. This result was extended to the Laplacian acting on differential forms in [7] and used in one of the proofs of the Ray-Singer conjecture; cf. [14].

A natural generalization of the Laplacian on $M$ is the connection (also called rough) Laplacian in whose definition the exterior $d$ is replaced by the covariant derivative $\nabla$ associated to a connection on a Hermitian vector bundle over $M$. Not much seems to be known about the spectra of such operators in general (but see [3). When the bundle is trivial of rank 1 (and usually in a noncompact setting) such connection Laplacians are also known and studied in the mathematical physics literature, e.g. [12, as magnetic Schrödinger operators.

In this paper we propose two definitions of combinatorial analogs of such connection Laplacians. Both rely on replacing the wedge product of differential forms by a graded commutative but nonassociative cup product on simplicial cochains, which is probably part of folklore and whose definition can be found for example in [4. In the first discretization scheme, which is applicable only for trivial bundles, we define combinatorial Laplacians corresponding to a coboundary operator twisted by a 1-cochain that is defined using the above cup product. The convergence of eigenvalues in degree zero (i.e. for the Laplacian acting on sections only, rather than on vector bundle valued forms) in this case is the content of Theorem 3.5. The second construction of discrete connection Laplacian uses the well-known fact, cf. 15, that any bundle with connection can be embedded into a trivial bundle

Received by the editors July 17, 2007, and, in revised form, September 13, 2007.

2000 Mathematics Subject Classification. Primary 58J50.

(c)2008 American Mathematical Society Reverts to public domain 28 years from publication 
in such a way that the connection is induced by the standard connection on the trivial bundle. Then the idea is to discretize this "classifying map". We establish the corresponding convergence of spectra in Theorem 4.4

We mention that our approach leads to a natural generalization of the discrete magnetic Laplacians on graphs studied in [19] and [13] to simplicial complexes. One can expect that an analogous convergence of spectrum distribution functions result for such twisted Laplacians can also be proved for noncompact universal covering manifolds, using the method of [5]. Finally, we note that a quite different discretization scheme for the degree zero case can be found in [10] and [11].

\section{The De Rham and Whitney maps}

In this note $M$ will always denote a closed oriented Riemannian manifold of dimension $N$. Let $\Omega(M)$ be the vector space of all smooth complex valued differential forms on $M$ and $L^{2}(\Lambda(M))$ be the space of square integrable forms on $M$ with respect to the volume element induced by the metric. Let $K$ be a smooth triangulation of $M$ and $C(K)$ be the vector space of (oriented) cochains of $K$ with complex coefficients. In what follows, $\|\omega\|$ will always denote the $L^{2}$-norm of the form $\omega$ and $\|c\|$, the norm of the cochain $c$ given by the canonical inner product on cochains with respect to which simplices are orthogonal.

Recall that for every $q>0$ the corresponding de Rham map $R^{K}: \Omega^{q}(M) \rightarrow$ $C^{q}(K)$ is given by $R^{K}(\omega)(\sigma)=\int_{\sigma} \omega$, where $\omega \in \Omega^{q}(M)$ and $\sigma$ is a $q$-simplex. When $q=0$ the de Rham map is defined to be simply the evaluation of a function on the 0 -skeleton of $K$.

The Whitney mapping $W^{K}$ goes in the reverse direction and is defined as follows. Let $\mu_{i}=\mu_{v_{i}}$ denote the barycentric coordinate function corresponding to a vertex $v_{i}$ and let $\sigma=\left[v_{0}, \ldots, v_{q}\right]$ be a $q$-simplex, where $q>0$. We set

$$
W^{K}(\sigma)=q ! \sum_{i=0}^{q}(-1)^{i} \mu_{i} d \mu_{0} \wedge \ldots \wedge d \mu_{i-1} \wedge d \mu_{i+1} \wedge \ldots \wedge d \mu_{q}
$$

If $q=0$ we set $W^{K}\left(v_{0}\right)=\mu_{0}$. This expression defines a map: $C^{q}(K) \rightarrow$ $L^{2}\left(\Lambda^{q}(M)\right)$. We note that the image of the Whitney map does not consist of smooth forms and summarize its basic properties in the following:

Proposition 2.1. (i) On the complement of the $(N-1)$-skeleton of $K$ one has $W^{K} d^{K}=d W^{K}$, where $d^{K}$ denotes the simplicial coboundary operator of $K$.

(ii) Let $j_{\sigma}$ denote the inclusion map $\sigma \hookrightarrow M$ and $j_{\sigma}^{*}$ the corresponding pull-back map on forms. Then the values of $j_{\sigma}^{*} W^{K} c$ (for $c$ an arbitrary cochain) depend only on the value of $c$ at $\sigma$. Therefore $R^{K}$ is well-defined on the image of $W^{K}$ and one has $R^{K} W^{K}=$ id on $C(K)$.

(iii) The support of $W^{K}(\sigma)$ is contained in the closed star of $\sigma$.

For proofs the reader is referred to [20] or [6].

The injectivity of the Whitney map allows us to define a new, metric dependent inner product on the space of cochains; namely, for $c_{1}, c_{2} \in C(K)$ we set $\left\langle c_{1}, c_{2}\right\rangle_{W}=$ $\left\langle W^{K} c_{1}, W^{K} c_{2}\right\rangle_{L^{2}}$. We will refer to this inner product as the Whitney inner product. 


\section{The TRIVIAL BUNDLE CASE}

3.1. A commutative cup product. We first let $K$ be any finite simplicial complex.

Definition 3.1 (4], 1], 21]). For two oriented simplices $\sigma_{1} \in C^{p}(K)$ and $\sigma_{2} \in$ $C^{q}(K)$, we set $\sigma_{1} \cup \sigma_{2}=0$ unless $\sigma_{1}$ and $\sigma_{2}$ meet at precisely one vertex and span a $(p+q)$-simplex $\tau$, in which case we define $\sigma_{1} \cup \sigma_{2}=\epsilon\left(\sigma_{1}, \sigma_{2}\right) \frac{p ! q !}{(p+q+1) !} \tau$, where the sign $\epsilon\left(\sigma_{1}, \sigma_{2}\right)= \pm 1$ is determined by the equation orientation $\left(\sigma_{1}\right)$.orientation $\left(\sigma_{2}\right)$ $=\epsilon\left(\sigma_{1}, \sigma_{2}\right)$.orientation $(\tau)$.

One checks that this defines a graded commutative nonassociative bilinear operation on $C(K)$ with respect to which the coboundary operator is a derivation.

In the case when $K$ is the underlying complex of a smooth triangulation of a compact manifold $M$, it was observed in [8] (see also [1] and [21] for more details) that this cup product admits an alternative description in terms of the Whitney and De Rham maps. Namely, for $a, b \in C(K)$ one has

$$
a \cup b=R^{K}\left(W^{K} a \wedge W^{K} b\right)
$$

Now let $A$ be a fixed real smooth 1-form. Consider the twisted exterior differential $d_{A}=d+i A \wedge$. and the corresponding Laplacian $\triangle_{A}=d_{A}^{*} d_{A}+d_{A} d_{A}^{*}$. We use the above to define discrete analogues of these operators.

Let $a$ be a fixed 1-cochain. Define the twisted coboundary operator associated to it as $d_{a}^{K}=d^{K}+i a \cup$., where $d^{K}$ is the usual coboundary of the simplicial complex $K$. Define the twisted discrete Laplacian by $\triangle_{a}^{K}=\left(d_{a}^{K}\right)^{*} d_{a}^{K}+d_{a}^{K}\left(d_{a}^{K}\right)^{*}$, where the adjoint is taken with respect to the inner product given by the Whitney map.

From now on we consider only triangulations $K$ which are subdivisions of a fixed triangulation and whose fullness is bounded away from 0 (see [20] or [7] for a definition). Then the cup product on cochains introduced above approximates the wedge product on forms according to the following result established in 21]. (The case when $\omega_{2}=1$ was proved in [6].)

Theorem 3.2. Let $\sigma$ be a simplex in $K, p$ - any point in the interior of $\sigma$ and $x_{1}, \ldots, x_{N}$-local coordinates around $\sigma$. Let $\omega_{1}, \omega_{2} \in \Omega(M)$. There exists a constant $C>0$ independent of $\omega_{1}, \omega_{2}, \sigma$ and $K$ such that

$$
\begin{aligned}
\mid W^{K}\left(R^{K} \omega_{1} \cup R^{K} \omega_{2}\right)(p) & -\left.\omega_{1} \wedge \omega_{2}(p)\right|_{p} \\
\leq & C h\left(\sup \left|\omega_{1}\right| \sup \left|\frac{\partial \omega_{2}}{\partial x_{i}}\right|+\sup \left|\omega_{2}\right| \sup \left|\frac{\partial \omega_{1}}{\partial x_{i}}\right|\right) .
\end{aligned}
$$

Here the suprema are taken over all partial derivatives and all points in $\sigma$.

Let us recall the definition of Sobolev spaces of vector bundle valued forms. Let $E$ be a Hermitian complex vector bundle over $M$ and let $\Omega(M, E)$ denote the smooth differential forms on $M$ with values in $E$. We fix a Hermitian connection on $E$ which defines a covariant differential $\nabla: \Omega^{*}(M, E) \rightarrow \Omega^{*+1}(M, E)$. Let $H^{r}(\Lambda(M, E))$ be the completion of $\Omega(M, E)$ with respect to the norm $\|\omega\|_{r}=\int_{M}\left|\left(1+\triangle_{E}\right)^{\frac{r}{2}} \omega\right|^{2} d v o l$, where $\triangle_{E}=\nabla^{*} \nabla+\nabla \nabla^{*}, \nabla^{*}$ being the formal adjoint of $\nabla$, is the connection Laplacian associated to $E$ and $\nabla$.

When $E$ is the trivial line bundle we write $H^{r}(\Lambda(M))$ for $H^{r}(\Lambda(M, E))$. In this case, integrating the estimate in Theorem 3.2 with $\omega_{2}=1$ and using the standard 
Sobolev inequality (see e.g. [16]), one obtains for every $r>\frac{N}{2}+1$ that

$$
\left\|W^{K} R^{K} \omega-\omega\right\| \leq C h\|\omega\|_{r} .
$$

3.2. Convergence of eigenvalues. We set $a=R^{K} A$ and use the above results to compare the operators $d_{A}$ and $d_{a}^{K}$. It turns out that the analogue of the identity from Proposition 2.1 (i) holds approximately in the twisted setting.

Lemma 3.3. For every $\omega \in \Omega(M)$ and every point $p \in M$ we have

$$
\begin{aligned}
& \left|W^{K} d_{a}^{K} R^{K} \omega-d_{A} W^{K} R^{K} \omega\right|_{p} \leq C_{A, \omega} h, \\
& \left|W^{K} d_{a}^{K} R^{K} \omega-W^{K} R^{K} d_{A} \omega\right|_{p} \leq C_{A, \omega} h,
\end{aligned}
$$

where $C_{A, \omega}$ is a constant depending only on $A$ and $\omega$ and their first derivatives.

Proof. In order to show (3.4), we write

$$
\begin{aligned}
W^{K} d_{a}^{K} R^{K} \omega-d_{A} W^{K} R^{K} \omega=W^{K}\left(R^{K} i A \cup R^{K} \omega\right)-i A \wedge W^{K} R^{K} \omega \\
=W^{K}\left(R^{K} i A \cup R^{K} \omega\right)-i A \wedge \omega-i A \wedge\left(W^{K} R^{K} \omega-\omega\right)
\end{aligned}
$$

and then apply Theorem 3.2 and the basic estimate (3.3). Similarly, observe that

$$
\begin{aligned}
W^{K} d_{a}^{K} R^{K} \omega-W^{K} R^{K} d_{A} \omega=W^{K}\left(R^{K} i A \cup R^{K} \omega\right)-W^{K} R^{K}(i A \wedge \omega) \\
=W^{K}\left(R^{K} i A \cup R^{K} \omega\right)-i A \wedge \omega+i A \wedge \omega-W^{K} R^{K}(i A \wedge \omega) .
\end{aligned}
$$

This easily implies (3.5).

Note that $d_{A}$ extends to a map $H^{1}(\Lambda(M)) \rightarrow L^{2}(\Lambda(M))$, also denoted by $d_{A}$.

Proposition 3.4. (i) For every $\omega \in \Omega(M)$ we have

$$
\left\|W^{K} d_{a}^{K} R^{K} \omega-d_{A} \omega\right\| \leq C_{A, \omega} h,
$$

where $C_{A, \omega}$ is a constant depending only on $A$ and $\omega$ and their derivatives.

(ii) For every $c \in C^{0}(K)$ we have

$$
\left\|W^{K} d_{a}^{K} c-d_{A} W^{K} c\right\| \leq C_{A}\left\|W^{K} c\right\|_{1} h .
$$

As above, $C_{A}$ depends only on $A$ and its derivatives of order up to $r$.

Proof. The estimate (3.6) is an easy consequence of (3.5). Observe that the image of $C^{0}(K)$ under the Whitney map consists of continuous piecewise linear functions and therefore is in $H^{1}(\Lambda(M))$. Denoting by $\|\cdot\|_{r}^{\sigma}$ for the $r$-th Sobolev norm on a closed $N$-simplex $\sigma$ computed using local coordinates in a neighborhood of $\sigma$, we obtain

$$
\begin{aligned}
& \left\|W^{K} d_{a}^{K} c-d_{A} W^{K} c\right\|^{2} \leq \sum_{\sigma \in K} \int_{\sigma}\left|W^{K} d_{a}^{K} c-d_{A} W^{K} c\right|^{2} d v o l \\
& \quad \leq \sum_{\sigma \in K} C_{A, h}^{\prime} h^{N+2}\left(\left\|W^{K} c\right\|_{r}^{\sigma}\right)^{2} \leq \sum_{\sigma \in K} C_{A} h^{2}\left(\left\|W^{K} c\right\|_{1}^{\sigma}\right)^{2} \leq C_{A} h^{2}\left\|W^{K}\right\|_{1}^{2} .
\end{aligned}
$$

Above we used the local estimate (3.4) with $\omega=W^{K} c$, a version of the Sobolev inequality which can be applied to each closed $N$-simplex (see e.g. [2, Theorem 3.9]) and the inequality $C_{A, h}^{\prime} \leq C_{A} h^{N}$. 
It is well-known that $\triangle_{A}$ with a domain of all smooth forms is an essentially selfadjoint elliptic operator on the compact manifold $M$ and hence its $L^{2}$ closure, denoted also by $\triangle_{A}$, has purely discrete spectrum. Let $\lambda_{0} \leq \lambda_{1} \leq \lambda_{2} \leq \ldots$ be the eigenvalues, repeated according to their multiplicities, of $\triangle_{A}$ acting on functions and let $\lambda_{0}^{K} \leq \lambda_{1}^{K} \leq \ldots \leq \lambda_{\operatorname{dim} C^{0}(K)}^{K}$ denote the eigenvalues of $\triangle_{a}^{K}$ acting on 0 -cochains. We have the following convergence of eigenvalues result.

Theorem 3.5. Let $j \leq \operatorname{dim} C^{0}(K)$. There exist positive constants $C_{A}$ and $C_{j, A}$ such that the following inequalities hold:

$$
\lambda_{j}^{K}-C_{j, A} h \leq \lambda_{j} \leq \frac{\left(\sqrt{\lambda_{j}^{K}}+C_{A} h\right)^{2}}{1-C_{A} h} .
$$

Proof. We begin by proving the second inequality. It follows from (3.7) that for every 0-cochain $c$ and some constant $C_{A} \geq 0$ depending only on $A$ and its first derivatives, one has

$$
\left\|W^{K} d_{a}^{K} c-d_{A} W^{K} c\right\| \leq C_{A} h\left(\left\|W^{K} c\right\|+\left\|d_{A} W^{K} c\right\|\right) .
$$

Thus we obtain

$$
\left\|W^{K} d_{a}^{K} c\right\| \geq\left(1-C_{A} h\right)\left\|d_{A} W^{K} c\right\|-C_{A} h\left\|W^{K} c\right\| .
$$

Using this, the min-max principle (see e.g. [18] gives

$$
\begin{aligned}
& \sqrt{\lambda_{j}^{K}}=\sup _{c_{1}, \ldots, c_{j-1} \in C^{0}(K)} \inf _{\substack{c \neq 0,\left\langle c, c_{k}\right\rangle=0, k=1,2, \ldots, j-1}} \frac{\left\|W^{K} d_{a}^{K} c\right\|}{\left\|W^{K} c\right\|} \\
& \geq \sup _{c_{1}, \ldots, c_{j-1} \in C^{0}(K)} \inf _{\substack{c \neq 0,\left\langle c, c_{k}\right\rangle=0, k=1,2, \ldots, j-1}}\left(\frac{\left(1-C_{A} h\right)\left\|d_{A} W^{K} c\right\|}{\left\|W^{K} c\right\|}-C_{A} h\right) \\
& \geq\left(1-C_{A} h\right) \sup _{f_{1}, \ldots, f_{j-1} \in W\left[C^{0}(K)\right]} \inf _{\substack{f \in W\left[C^{0}(K)\right] \backslash\{0\},\left\langle f, f_{k}\right\rangle=0, k=1, \ldots, j-1}} \frac{\left\|d_{A} f\right\|}{\|f\|}-C_{A} h \\
& \geq\left(1-C_{A} h\right) \sqrt{\lambda_{j}}-C_{A} h .
\end{aligned}
$$

Hence the second inequality holds.

To establish the first inequality, denote by $V_{j}$ the vector space spanned by the first $j$ eigenfunctions of $\triangle_{A}$. Then it follows from (3.6) that for each $f \in V_{j} \backslash\{0\}$ and for some constant $C_{j, A}$ depending only on $j$ and $A$ we have

$$
\left|\frac{\left\langle W^{K} d_{a}^{K} R^{K} f, W^{K} d_{a}^{K} f\right\rangle}{\left\langle W^{K} R^{K} f, W^{K} R^{K} f\right\rangle}-\frac{\left\langle d_{A} f, d_{A} f\right\rangle}{\langle f, f\rangle}\right| \leq C_{j, A} h .
$$

Then the proof proceeds in exactly the same fashion as in [6, Theorem 5.7].

\section{The General Bundle CASE}

4.1. The universal connection. Now let $E$ be a Hermitian complex vector bundle of rank $d$ over $M$. We denote the space of $L^{2}$ forms with values in $E$ defined using the inner product on $E$ by $L^{2}(\Lambda(M), E)$ and fix a Hermitian connection on $E$ which defines a covariant differential $\nabla: \Omega^{*}(M, E) \rightarrow \Omega^{*+1}(M, E)$. We shall need the following theorem on the existence of universal connections due to Narasimhan and Ramanan [15], in the form proved in the appendix of [17]. 
Theorem 4.1. There exist a trivial Hermitian bundle $V$ and an isometric embedding of bundles $i: E \hookrightarrow V$ such that $\nabla=i^{*} \circ d \circ i$, where $i^{*}$ denotes the fiberwise adjoint taken with respect to the corresponding inner products.

Let us briefly recall how the Narasimhan-Ramanan theorem is proved, as the construction will be needed in the sequel. One first shows that the statement is true for trivial bundles. Then one chooses a finite open cover $\left\{U_{l}\right\}$ of $M$ such that $E$ restricted to every $U_{l}$ is trivial and is a partition of unity $\left\{\psi_{l}\right\}$ subordinate to this cover such that $\sum_{l} \psi_{l}^{2}=1$. If $i_{l}$ denotes the already constructed isometric embedding from $\left.E\right|_{U_{l}}$ to a trivial bundle $V_{l}$, one defines

$$
i=\sum_{l} i_{l} \psi_{l}: E \rightarrow V
$$

where $V=\bigoplus_{l} V_{l}$.

In what follows, we will assume that we have fixed a triangulation $K_{0}$ fine enough so that there exists a finite open cover $\left\{U_{l}\right\}$ as above with the additional properties that (1) each $\bar{U}_{l}$ is a subcomplex of $K_{0}$, and (2) $E$ restricted to $\bar{U}_{l}$ is trivial, and we will consider only subdivisions $K$ of $K_{0}$. We will also assume that the map $i$ is constructed using such an open cover.

4.2. Twisted de Rham and Whitney maps. We first define the space of cochains on which the discrete connection Laplacian will act. We denote the induced map from $L^{2}(\Lambda(M), E)$ to $L^{2}(\Lambda(M), V)$ also by $i$. We would like to define cochains on $K$ with values in the bundle $E$. To this end, fix a reference point $p_{\sigma}$ in the interior of each simplex $\sigma$ in $K$.

Definition 4.2. Let $C(K, E)$, the twisted cochains with values in $E$, denote the set of all maps from the set of all simplices in $K$ to the total space of $E$ which assign to each simplex $\sigma$ a vector in the fibre $E_{p_{\sigma}}$.

This is a vector space with an inner product induced by the Hermitian structure on $E$. The restriction of $i$ to fibers defines a map, denoted by $i^{K}$, from $C(K, E)$ to $C(K, V)$ which we identify with the cochains taking values in $\mathbb{C}^{n}$, where $n$ is the rank of the trivial bundle $V$.

We shall also need the following notation. Let $I_{l}^{K}: C\left(\overline{U_{l}}, \mathbb{C}^{d}\right) \rightarrow C\left(\overline{U_{l}}, \mathbb{C}^{n}\right)$ be the operator given by $\left(I_{l}^{K} c\right)_{p}=\sum_{s} R^{K}\left(i_{p s}\right) \cup c_{s}$. Here $i_{p s}$ are the entries of the matrix of $i$ and $c_{p}$ the components of the vector valued cochain $c$. We define the operator $\Psi_{l}^{K}$ by $\Psi_{l}^{K} c_{p}=R^{K}\left(\psi_{l}\right) \cup c_{p}$. Finally we define $I^{K}: C(K, E) \rightarrow C(K, V)$ by $I^{K} c=\sum_{l} I_{l}^{K} \Psi_{l}^{K} c$. Now define an operator acting on $C(K, E)$, the discretization of $\nabla$, by setting $\nabla^{K}=\left(I^{K}\right)^{*} d^{K} I^{K}$.

In order to compare $\nabla^{K}$ and $\nabla$ we have to introduce the appropriate twisted Whitney and de Rham maps. We set $\widetilde{W}^{K}=i^{*} W^{K} I^{K}$ and $\widetilde{R}^{K}=\left(I^{K}\right)^{*} R^{K} i$. Here $W^{K}$ and $R^{K}$ are the usual Whitney and de Rham maps, but now acting between the spaces of vector valued cochains and differential forms, $C(K, V)$ and $L^{2}(\Lambda(M), V)$, respectively.

It is easily seen that $\widetilde{W}^{K}$ is injective on 0-cochains. More generally, we have:

Lemma 4.3. For sufficiently fine triangulations $K$ the map $\widetilde{W}^{K}$ is injective on $C^{q}(K, E)$ for all $q$. 
The proof will be given in the next section. We can now define the corresponding Whitney inner product on $C(K, E)$ as in Section 2 and the discrete connection Laplacian $\triangle_{E}^{K}=\left(\nabla^{K}\right)^{*} \nabla^{K}+\nabla^{K}\left(\nabla^{K}\right)^{*}$, where the adjoint is taken with respect to this inner product. Let $\lambda_{0} \leq \lambda_{1} \leq \lambda_{2} \leq \ldots$ denote the eigenvalues repeated according to their multiplicities, of (the $L^{2}$ closure of) the connection Laplacian $\triangle_{E}=\nabla^{*} \nabla+\nabla \nabla^{*}$ acting on sections of $E$, and let $\lambda_{0}^{K} \leq \lambda_{1}^{K} \leq \ldots \leq \lambda_{\operatorname{dim} C^{0}(K, E)}^{K}$ denote the eigenvalues of $\triangle_{E}^{K}$ acting on 0 -cochains with values in $E$. We have the following convergence of spectra result.

Theorem 4.4. There exist positive constants $C^{i}$ and $C_{j}^{i}$ such that the following inequalities hold for $j \leq \operatorname{dim} C^{0}(K, E)$ :

$$
\lambda_{j}^{K}-C_{j}^{i} h \leq \lambda_{j} \leq \frac{\left(\sqrt{\lambda_{j}^{K}}+C^{i} h\right)^{2}}{1-C^{i} h}
$$

\section{Proof of Theorem 4.4}

5.1. Estimate from below. In this subsection we estimate from below the eigenvalues of the connection Laplacian in terms of the eigenvalues of its discretizations; i.e. we prove the first inequality in Theorem 4.4. We denote the projection $i i^{*}$ by $P$ and introduce the "almost" projection operators $P^{K}=I^{K}\left(I^{K}\right)^{*}$. Our next lemma states that the Whitney and de Rham maps approximately interwine $P$ and $P^{K}$.

Lemma 5.1. For $r>\frac{N}{2}+2$, every form $\omega \in \Omega(M, V)$ and every vector valued function $f \in \Omega^{0}(M, V)$, one has

$$
\begin{gathered}
\left\|W^{K} P^{K} R^{K} \omega-W^{K} R^{K} P \omega\right\| \leq C_{i} h\|w\|_{r}, \\
\left\|W^{K} P^{K} R^{K} \omega-P W^{K} R^{K} \omega\right\| \leq C_{i} h\|w\|_{r}, \\
P^{K} R^{K} f=R^{K} P f .
\end{gathered}
$$

Here $C_{i}$ denotes a constant depending only on the map $i$ and its derivatives.

Proof. We first show that (5.1) holds. In the course of the proof, various constants depending on $i$ will all be denoted by $C_{i}$.

We first assume that the bundle $E$ is trivial and identify $C(K, E)$ with $C\left(K, \mathbb{C}^{d}\right)$ and $\Omega(M, E)$ with $\Omega\left(M, \mathbb{C}^{d}\right)$. Let $\sigma$ be a $q$-simplex in $K$ and let $\omega \in \Omega^{q}(M, E)$. We write $R^{K}$ (respectively $W^{K}$ ) for both de Rham (Whitney) maps defined on $C\left(K, \mathbb{C}^{d}\right)$ and $C(K, V)$ (respectively on $\Omega\left(M, \mathbb{C}^{d}\right)$ and $\Omega(M, V)$ ), and observe that, in view of [7, Lemma 7.22], for every $c \in C(K, V)$ one has

$$
C_{1} h^{N-2 q}\|c\|^{2} \leq\left\|W^{K} c\right\|^{2} \leq C_{2} h^{N-2 q}\|c\|^{2}
$$

where the constants $C_{1}$ and $C_{2}$ do not depend on $K$. From the definitions we have $I^{K} R^{K} \omega-R^{K} i \omega=\sum_{s}\left(R^{K}\left(i_{p s}\right) \cup R^{K} \omega_{s}-R^{K}\left(i_{p s} \omega_{s}\right)\right)$. Thus, combining (3.3), (3.2) and (5.4) we conclude that

$$
\begin{aligned}
& \left\|W^{K} I^{K} R^{K} \omega-W^{K} R^{K} i \omega\right\| \\
& \quad \leq \sum_{s}\left(\left\|W^{K}\left(R^{K}\left(i_{p s}\right) \cup R^{K} \omega_{s}-i_{p s} \omega_{s}\right)\right\|+\left\|W^{K} R^{K} i_{p s} \omega_{s}-i_{p s} \omega_{s}\right\|\right) .
\end{aligned}
$$

Now (5.4) implies that

$$
\left\|I^{K} R^{K} \omega-R^{K} i \omega\right\|^{2} \leq C_{i} h^{2 q-N+2}\|\omega\|_{r}^{2} .
$$


It is easy to check that $\left(\left(I^{K}\right)^{*} c\right)_{p}=\sum_{s} R^{K}\left(i_{p s}^{*}\right) c_{s}$. Hence we also have

$$
\left\|\left(I^{K}\right)^{*} R^{K} \omega-R^{K} i^{*} \omega\right\|^{2} \leq C_{i} h^{2 q-N+2}\|\omega\|_{r}^{2} .
$$

Next, observe that the operators $I^{K}$ are uniformly bounded with respect to $K$. Indeed, from the combinatorial definition of the cup product and the mean value theorem one easily obtains

$$
\left\|i^{K} R^{K} \omega-I^{K} R^{K} \omega\right\|^{2} \leq C_{i} h^{2 q-N+2}\|\omega\|_{r}^{2}
$$

and $i^{K}$ is certainly uniformly bounded. We use this fact in the following estimate:

$$
\begin{aligned}
& \left\|P^{K} R^{K} \omega-R^{K} P \omega\right\|^{2} \\
\leq & \left\|I^{K}\left(I^{K}\right)^{*} R^{K} \omega-I^{K} R^{K} i^{*} \omega\right\|^{2}+\left\|I^{K} R^{K} i^{*} \omega-R^{K} i i^{*} \omega\right\|^{2} \leq C_{i} h^{2 q-N+2}\|\omega\|_{r}^{2} .
\end{aligned}
$$

Applying (5.4) again, we see that

$$
\left\|W^{K} P^{K} R^{K} \omega-W^{K} R^{K} P \omega\right\| \leq C_{i} h\|\omega\|_{r} .
$$

Now let $E$ be an arbitrary bundle. We use the open cover $\left\{U_{l}\right\}$ and the partition of unity $\left\{\psi_{l}\right\}$ with the properties described after Theorem 4.1 and define $P_{l}=i_{l} i_{l}^{*}$. Then, using (4.1), we find that $P=\sum_{l, k} i_{l} \psi_{l} \psi_{k} i_{k}^{*}=\sum_{l} i_{l} \psi_{l}^{2} i_{l}^{*}$. On the other hand, $P^{K}$ is not equal to $\sum_{l} I_{l}^{K}\left(\Psi_{l}^{K}\right)^{2}\left(I_{l}^{K}\right)^{*}$, due to the nonassociativity of our cup product. However, according to Theorem 3.2 this product is approximately associative; therefore we can replace $P^{K}$ by $\sum_{l} I_{l}^{K}\left(\Psi_{l}^{K}\right)^{2}\left(I_{l}^{K}\right)^{*}$ in the following estimate:

$$
\begin{aligned}
&\left\|P^{K} R^{K} \omega-R^{K} P \omega\right\| \\
&\left.\leq \sum_{l}\left(\left\|\sum_{l}^{K}\left(\Psi_{l}^{K}\right)^{2}\left(\left(I_{l}^{K}\right)^{*} R^{K} \omega-I_{l}^{K}\left(\Psi_{l}^{K}\right)^{2}\left(I_{l}^{K}\right)^{*} \omega\right)\right\|+\left\|R^{K} \omega-R^{K} i_{l} \psi_{l}^{2} i_{l}^{*} \omega\right\|+O\left(\Psi_{l}^{K}\right)^{2} R^{K} i_{l}^{*} \omega-R^{K} \psi_{l}^{2} i_{l}^{*} \omega\right) \|\right) \\
&+\sum_{l}\left\|\left(R^{K} i_{l}-I_{l}^{K} R^{K}\right) \psi_{l}^{2} i_{l}^{*} \omega\right\|+O(h) \leq C_{i} h^{q-\frac{N}{2}+1}\|\omega\|_{r}^{2} .
\end{aligned}
$$

Above we used (5.5) and (5.6) already proved over each $\bar{U}_{l}$, and the inequality

$$
\left\|\left(\Psi_{l}^{K}\right)^{2} R^{K} \omega-R^{K} \psi_{l}^{2} \omega\right\|^{2} \leq C_{i} h^{2 q-N+2}\|\omega\|_{r}^{2},
$$

which is proved in exactly the same manner as (5.5). Thus (5.1) is shown to hold for arbitrary $E$.

The estimate (5.2) can easily be derived from (5.1). Finally, the equality (5.3) follows directly from the definitions.

We use this lemma to deduce that the combinatorial connection approximates the smooth one in the appropriate sense.

Proposition 5.2. For $r>\frac{N}{2}+1$, every section $f \in \Omega^{0}(M, E)$ and every 0 -cochain $c \in C(K, E)$ one has

$$
\left\|\widetilde{W}^{K} \nabla^{K} \widetilde{R}^{K} f-\nabla f\right\| \leq C_{1}^{i} h\|f\|_{r}
$$


Proof. We estimate as follows, using (5.3), (5.2) and (3.3):

$$
\begin{aligned}
& \left\|\widetilde{W^{K}} \nabla^{K} \widetilde{R}^{K} f-\nabla f\right\|=\left\|i^{*} W^{K} P^{K} d^{K} P^{K} R^{K} i f-i^{*} d i f\right\| \\
& =\left\|i^{*} W^{K} P^{K} R^{K} d i f-i^{*} d i f\right\| \leq\left\|\left(W^{K} P^{K} R^{K}-P^{K} W^{K} R^{K}\right) d i f\right\| \\
& \quad+\| W^{K} R^{K} \text { dif }-\operatorname{dif}\left\|\leq C_{1}^{i} h\right\| f \|_{r} .
\end{aligned}
$$

The first inequality in Theorem 4.4 follows as in the proof of Theorem 3.5 .

5.2. Estimate from above. In this subsection we estimate from above the eigenvalues of the connection Laplacian in terms of the eigenvalues of its discretizations, i.e. we prove the second inequality in Theorem 4.4. This will follow from Proposition 5.2. We will need the following strengthened version of Lemma 5.1.

Lemma 5.3. For $r>\frac{N}{2}+2$ and every form $\omega \in \Omega(M, V)$ one has

$$
\begin{aligned}
& \left\|d W^{K} P^{K} R^{K} \omega-d W^{K} R^{K} P \omega\right\| \leq C_{i} h\|w\|_{r}, \\
& \left\|d W^{K} P^{K} R^{K} \omega-d P W^{K} R^{K} \omega\right\| \leq C_{i} h\|w\|_{r} .
\end{aligned}
$$

Above, $C_{i}$ is a constant depending only on the map $i$ and its derivatives.

Proof. The argument is similar to the proof of Lemma 5.1 but now one has to use in addition the derivation property of $d^{K}$. The details are omitted.

We now use this lemma to derive the following proposition, which is the analog of part (ii) of Proposition 3.4 in this situation.

Proposition 5.4. For every 0 -cochain $c \in C(K, E)$ one has

$$
\left\|\widetilde{W}^{K} \nabla^{K} c-\nabla \widetilde{W}{ }^{K} c\right\| \leq C^{i} h\left(\left\|\widetilde{W}^{K} c\right\|+\left\|\nabla \widetilde{W}^{K} c\right\|\right) .
$$

Proof. We first prove the analogous estimate in the case when $c=\widetilde{R}^{K} f$ for some smooth section $f$. Using (5.2), (5.10) and (5.3) one has for $r$ large enough:

$$
\begin{gathered}
\left\|\widetilde{W^{K}} \nabla^{K} \widetilde{R}^{K} f-\nabla \widetilde{W^{K}} \widetilde{R}^{K} f\right\|=\left\|i^{*} W^{K} P^{K} R^{K} d i f-i^{*} d P W^{K} P^{K} R^{K} i f\right\| \\
\leq\left\|\left(W^{K} P^{K} R^{K}-P W^{K} R^{K}\right) d i f\right\|+\left\|d W^{K} P^{K} R^{K} i f-d P W^{K} R^{K} i f\right\| \leq C_{2}^{i} h\|f\|_{r} .
\end{gathered}
$$

Now we note that (5.3) implies that $c=\widetilde{R}^{K} \widetilde{W}^{K}$ and proceed exactly as in the proof of part (ii) of Proposition 3.4. The only difference now is that the image of $\widetilde{W}^{K}$ does not consist of piecewise linear sections of $E$ anymore. However one can pass from the $r$-th to the first Sobolev norm on each $N$-simplex simply by repeated use of the Leibniz rule.

It remains to prove Lemma 4.3. Observe that according to (5.7) it suffices to prove that $i^{*} W^{K} i^{K}$ is injective.

Suppose that $c_{0} \in C^{q}(K, E)$ is a nonzero cochain supported on a single $q$-simplex $\sigma$ with reference point $p_{\sigma}$. A direct computation in local coordinates shows that $i^{*} W^{K} i^{K} c_{0}$ is not identically zero. Now let $c$ be an arbitrary nonzero cochain and suppose that $i^{*} W^{K} i^{K} c=0$. Suppose also that $c$ is not zero at a simplex $\sigma$. Then Proposition 2.1 will imply that $j_{\sigma}^{*}\left(i^{*} W^{K} i^{K} c\right)=i^{*} j_{\sigma}^{*}\left(W^{K} i^{K} c\right)$ is not zero, a contradiction. 


\section{ACKNOWLEDGEMENT}

This work is part of the author's Ph.D. thesis completed under the guidance of Professor Józef Dodziuk, to whom many thanks are due for his constant support.

\section{REFERENCES}

[1] Adams, D.: $R$-torsion and linking numbers from simplicial Abelian gauge theories. Preprint, hep-th/9612009.

[2] Agmon, S.: Lectures on elliptic boundary value problems. Van Nostrand Co., Princeton, 1965. MR0178246 (31:2504)

[3] Ballmann, W., Brünning, J. and Carron, G.: Eigenvalues and holonomy. Int. Math. Res. Not. 1 (2003), 657-665. MR1951401 (2003j:53068)

[4] Birmingham, D. and Rakowski, M.: A star product in lattice gauge theory. Phys. Lett. B 299 (1993), 299-304. MR 1201283 (94e:81225)

[5] Carey, A., Couhlon, T., Mathai, V, and Phillips, J.: Von Neumann spectra near the spectral gap. Bull. Sci. Math. 122 (1998), no. 3, 203-242. MR.1621581 (99g:58128)

[6] Dodziuk, J.: Finite difference approach to the Hodge theory of harmonic forms. Amer. J. Math. 98 (1976), 79-104. MR0407872 (53:11642)

[7] Dodziuk, J. and Patodi, V.K.: Riemannian structures and triangulations of manifolds. J. Indian Math. Soc. 40 (1976), 1-52. MR0488179(58:7742)

[8] Dupont, J.: Simplicial de Rham cohomology and characteristic classes of flat bundles. Topology 15 (1976), no. 3, 233-245. MR0413122 (54:1243)

[9] Eckmann, B.: Harmonische Funktionen und Randwertaufgaben in einem Komplex. Comment. Math. Helv. 17 (1945), 240-255. MR0013318(7:138f)

[10] Mantuano, T.: Discretization of compact Riemannian manifolds applied to the spectrum of Laplacian. Ann. Global Anal. Geom. 27 (2005), 33-46. MR2130531 (2006e:58046)

[11] Mantuano, T.: Discretization of vector bundles and rough Laplacian. Preprint, 2006.

[12] Mathai, V. and Shubin, M.: Semiclassical asymptotics and gaps in the spectra of magnetic Schrödinger operators. Geom. Dedicata 91 (2002), 155-173. MR1919898 (2004f:58040)

[13] Mathai, V. and Yates, S.: Approximating spectral invariants of Harper operators on graphs. J. Funct. Anal. 188 (2002), no. 1, 111-136. MR.1878633 (2002k:47070)

[14] Müller, W.: Analytic torsion and R-torsion of Riemannian manifolds. Adv. in Math. 28 (1978), no. 3, 233-305. MR498252 (80j:58065b)

[15] Narasimhan, M. and Ramanan, S.: Existence of universal connections. Amer. J. Math. 83 (1961), 563-572. MR0133772 (24:A3597)

[16] Narasimhan, R.: Analysis on Real and Complex Manifolds. North-Holland, Amsterdam, 1968. MR0251745(40:4972)

[17] Quillen, D.: Superconnection character forms and the Cayley transform. Topology 27 (1988), no. 2, 211-238. MR 948184 (89j:58134)

[18] Reed, M. and Simon, B.: Methods of Modern Mathematical Physics. IV. Analysis of Operators. Academic Press, New York-London, 1978. MR0493421 (58:12429c)

[19] Sunada, T.: A discrete analogue of periodic magnetic Schrödinger operators. Contemp. Math. 173 (1994), 283-299. MR1298211 (95i:58185)

[20] Whitney, H.: Geometric Integration Theory. Princeton University Press, Princeton, 1957. MR0087148(19:309c)

[21] Wilson, S.: Geometric structures on the cochains of a manifold. Preprint, Math.GT/0505227.

Department of Mathematics and Computer Science, Lehman College of CUNy, 250

Bedford Park Boulevard West, Bronx, New York 10468

E-mail address: szahariev@gc.cuny.edu 